\title{
Report to the Public in the United Kingdom - the Basic Understanding of Bovine Spongiform Encephalopathy (BSE)
}

\section{Shin-Jye Lee ${ }^{1}$; Hsueh-Cheng Liu ${ }^{1}$;Ching-Hsun Tseng ${ }^{2}$; Ying-Yi Chou ${ }^{1}$}

Affiliation: Institute of Management of Technology, National Chiao Tung University, Taiwan ${ }^{1}$; Department of Computer Science, University of Manchester, United Kingdom²

\author{
E-mail: camhero@gamil.com ${ }^{1}$; stdm11528@gmail.com ${ }^{2}$; hank131415go61@gmail.com ${ }^{3}$;
} shiaopooh1811@gmail.com ${ }^{4}$

DOI: 10.26821/IJSRC.9.4.2021.9416

\begin{abstract}
Bovine spongiform encephalopathy (BSE) brought a great fear of beef to the public in the United Kingdom since 1996. In order to positively realize the essence of this problem, the correct knowledge of the bovine spongiform encephalopathy (BSE) is essentially necessary, including the basic background, origin, events, policies and legislation relating to the bovine spongiform encephalopathy (BSE) worldwide. So far as the understanding of the bovine spongiform encephalopathy (BSE) to the public in the United Kingdom is concerned, correctly choose the low BSEinfected potential beef is especially important in the daily life. Therefore, an easy-understanding, clear, and basic knowledge of bovine spongiform encephalopathy $(B S E)$ is the core of problem definition in this report.
\end{abstract}

Keywords: Bovine Spongiform Encephalopathy

\section{INTRODUCTION}

Bovine spongiform encephalopathy (BSE) brought a great fear of beef to the public in the United Kingdom since 1996. In order to positively realize the essence of this problem, the correct knowledge of the bovine spongiform encephalopathy (BSE) is essentially necessary, including the basic background, origin, events, policies and legislation relating to the bovine spongiform encephalopathy (BSE) worldwide. So far as the understanding of the bovine spongiform encephalopathy (BSE) to the public in the United Kingdom is concerned, correctly choose the low BSEinfected potential beef is especially important in the daily life. Therefore, an easy-understanding, clear, and basic knowledge of bovine spongiform encephalopathy (BSE) is the core of problem definition in this report.

\section{BACKGROUND OF BOVINE} \section{SPONGIFORM ENCEPHALOPATHY}

The first bovine spongiform encephalopathy (BSE) was diagnosed in November 1986 at the Central Veterinary Laboratory in the England, and the first case of bovine spongiform encephalopathy (BSE) was therefore identified in the United Kingdom in 1986. With the unexpected occurrence of bovine spongiform encephalopathy (BSE), bovine spongiform encephalopathy (BSE)widely spread to European continent, North America and Asia, and its huge infection caused countries in these continents becoming quarantine areas in the last two decades [1].As mentioned above, bovine spongiform encephalopathy (BSE) is a relatively new disease of cattlefirstly recognized and defined in the United Kingdom in November 1986. Over the following few years, the bovine spongiform encephalopathy (BSE)epidemic grew considerably and speedily affected lots of countrieswith different degrees. Meanwhile, the infection of bovine spongiform encephalopathy (BSE) reached its peak in 1992, whilst 36,680 cases were confirmed in the United Kingdom.After the peak of bovine spongiform encephalopathy (BSE) in 1992, the bovine spongiform encephalopathy (BSE)epidemic wad going to a steady decrement. According to the infection of bovine spongiform encephalopathy (BSE), bovine spongiform encephalopathy(BSE) occurs either in adult animals or in both genders, typically in animals aged five years and more. Also, the clinical disease usually lasts for 
ISSN-2347-9698

Volume 9 Issue 4 April 2021

several weeks and it is invariably progressive and fatal[1].

Bovine spongiform encephalopathy (BSE) is commonly known as mad cow disease. The origin of BSE epidemic is considered that resulted from feeding cattle infectious meat and bone meal (MBM) for decreasing the feeding costs and most of the meat and bone meal (MBM) is produced by the rendering of carcases of infected cattle [2]. Based on biological sciences, bovine spongiform encephalopathy (BSE) is a fatal neurodegenerative disease in cattle that causes a spongy degeneration in the brain and spinal cord, as shown in Figure $1 \&$ Figure 2. Whilst the cattle have been infected bovine spongiform encephalopathy (BSE), the primary symptoms comprise weakening the brain functions of cattle, insanity, ataxia, and then death. Also, the neurological disease in which affected animals show the following signs, including changes in mental state, abnormalities of posture and movement and of sensation[1]. In addition, the bovine spongiform encephalopathy(BSE) has a long incubation period, and it is generally about four years.It usually affects adult cattle at a peak age, occurring at the onset of four to five years in particular. Moreover, the infectious agent in the bovine spongiform encephalopathy(BSE) is considered to be a specific type of misfoldedprotein called a prion. The bovine spongiform encephalopathy(BSE) is therefore believed to belinked to the variant Creutzfeldt-Jakob disease (vCJD), one of fatal transmissible spongiform encephalopathy (TSE) diseases found in humans, andthe first human infected by variant Creutzfeldt-Jakob disease (vCJD) was believed to be caused by bovine spongiform encephalopathy(BSE) in 1996. Basically, prion proteins carry the disease between individuals and cause deterioration of the brain, and BSE is a type of transmissible spongiform encephalopathy (TSE). The relationship of the infective bovine spongiform encephalopathy(BSE) agent and variant CreutzfeldtJakob disease (vCJD) in humans is not completely understood and no direct correlation has been confirmed.However, a strong association exists between humans infected with variant CreutzfeldtJakob disease (vCJD) and exposure to BSE-infected products[3].

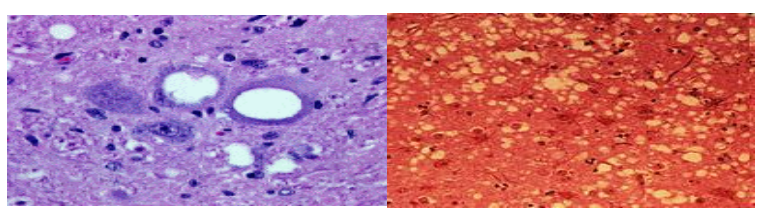

Fig 1\& 2. Infected brain tissue (Source: Academia Sinica
Taiwan \& Animal and Plan Health Inspection Service)

\section{Events, Policies, and Legislations Relating to the Bovine Spongiform Encephalopathy(BSE) Worldwide}

Since the first bovine spongiform encephalopathy (BSE) had been identified in the United Kingdom in March 1996, the enforcement of legislative controls to eliminate risk of bovine spongiform encephalopathy (BSE) pandemic include: (1)Meat and Bone Meal (MBM) feed ban; (2)Bovine Spongiform Encephalopathy (BSE) testing: cattle aged over 48 months (30 months for non-EU member states) must test negative before permitted into food chain; (3)Specific Risk Material (SRM) control: bypass of parts of cattle most likely to carry BSE infection; (4)Ban on production of mechanical separated meat (MSM) from all ruminant bones. Further, the events and corresponding policies relating to the bovine spongiform encephalopathy (BSE) worldwide can be described as follows:

- In March 1996, the first human infected by variant Creutzfeldt-Jakob disease (vCJD) was believed to be caused by the bovine spongiform encephalopathy(BSE) in the United Kingdom, and it had been proved that bovine spongiform encephalopathy(BSE) has relationships with transmissible spongiform encephalopathy of human being. Meanwhile, the worst country getting involved in bovine spongiform encephalopathy(BSE) is United Kingdom. The bovine spongiform encephalopathy(BSE) cases found in the United Kingdom took more than $95 \%$ in the worldwide bovine spongiform encephalopathy(BSE) cases, and the bovine spongiform encephalopathy(BSE) crisis therefore led the European Union to strictly ban exports of British beef. On 27th March 1996 the European Commission prohibited all United Kingdom exports of beef and cattle and their byproducts to all other European Union member states and the rest of the world.

- In September 2001, the first bovine spongiform encephalopathy(BSE) case had been found in Japan, and the Japanese government was thus forcedly banned exports of Japanese beef.

- In December 2003, the bovine spongiform encephalopathy(BSE) had been found in America and Canada. Also, America is one of primary countries exporting the most beef 
ISSN-2347-9698

Volume 9 Issue 4 April 2021

worldwide, and some countries which imported a lot of American beefs were hence unfortunately importing bovine spongiform encephalopathy(BSE) as well. Afterwards, several countries immediately banned importing American beef.

- In 2005, due to the strong exporting policy of American beef, several countries conditionally imported American beef, and the condition was built on the importing American beef should be absolutely less than 30 moths' aged and boneless beef.

- In November 2006, North Ireland made a mistake in exporting a 54 months' aged cow without export quarantine, and with the amount of beef productions to the United Kingdom, Italy and Spain. Afterwards, all of the beef productions exported from North Ireland were reclaimed immediately. In the general domestic policy of bovine spongiform encephalopathy(BSE), only 30 months' aged cow and less can be exempted from export quarantine.

- In November 2006, 30 bovine spongiform encephalopathy(BSE) cases had been found in Japan, and the infected cattle were considered by feeding infectious meat and bone meal (MBM). In addition, two variant Creutzfeldt-Jakob disease (vCJD) cases had been found in Netherland.

Further, a geographical distribution of countries that reported at least one bovine spongiform encephalopathy(BSE) confirmed case since 1989 can be displayed in Figure 3.

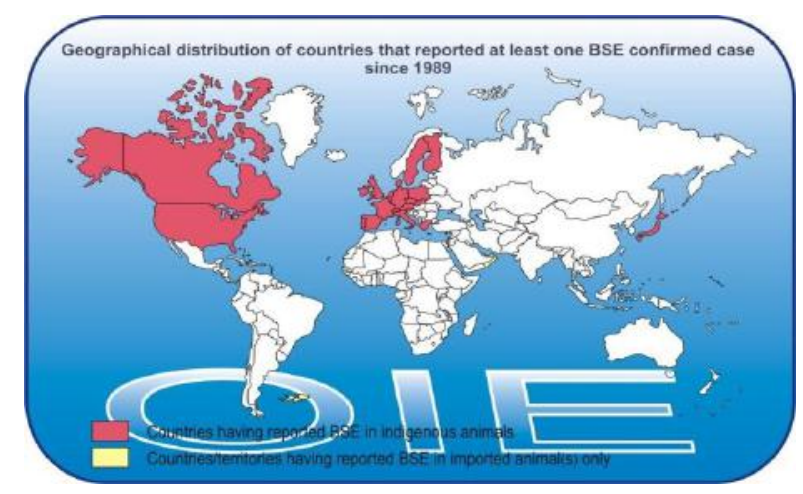

Fig 3. Geographical distribution of countries of bovine spongiform encephalopathy (BSE) since 1989 (Source: World Organization for Animal Health (OIE), www.oie.int)

\section{HOW TO CHOOSE THE LOW BSE- INFECTED POTENTIAL BEEF?}

A strong association exists between humans infected with variant Creutzfeldt-Jakob disease (vCJD) and exposure to BSE-infected products [3]. To avoid infecting variant Creutzfeldt-Jakob disease (vCJD) as far as possible, correctly choosing the low BSEinfected potential beef and keeping away the high BSE-infected potential beef are therefore completely important. So far as the level of BSE-infected potential is concerned, the level of BSE-infected potential of beef and beef productions depends on the part of the cattle. The lowest BSE-infected potential beef is boneless beef, and the highest BSE-infected potential beef is brain organization, the trigeminal nerve, spinal cord, ileum, and eyeball. Moreover, the BSE-infected probability of boneless beef is $7.18 \times 10^{-12}$, that of beef with bone is $2.72 \times 10^{-11}$, that of cow internal organs is $1.5 \times 10^{-10}$, and that of Cow internal organs is $5.77 \times 10^{-10}$. Therefore, the possibility of being infected bovine spongiform encephalopathy (BSE) by eating boneless beef is $7.18 \times 10^{-12}$. Based on the possibility of being infected bovine spongiform encephalopathy (BSE) by eating boneless beef ( $7.18 \times 10^{-12}$ ), the possibility of being infected bovine spongiform encephalopathy (BSE) by eating beef with bone is 3.7 times that of boneless beef, the possibility of being infected bovine spongiform encephalopathy (BSE) by eating cow internal organs is 20 times that of boneless beef, and the possibility of being infected bovine spongiform encephalopathy (BSE) by eating beef mince is 700 times that of boneless beef [4]. Thus, the level of BSE-infected potential of beef in a low-tohigh order is:

Boneless Beef < Beef with Bone < Cow Internal Organs $<$ Beef Mince

In the light of the above relationship of BSE-infected potential, the beef mince is considered the highest-risk level of BSE-infected product in all of beef productions, because the beef mince is generally pounded by 10,000 pounds beef in each time in America. Furthermore, there is not any definiteguarantee that this huge amount of beef before pounding is healthy and whether the source of beef is from non-infected area. Further, correctly choosing the relevant productions from the non-infected areas and keeping away the productions from the infected areas 
is one of positive ways to avoid bovine spongiform encephalopathy(BSE).

\section{CONCLUSION}

Today, the research of the bovine spongiform encephalopathy (BSE) is quite mature, and the knowledge of the bovine spongiform encephalopathy (BSE) is therefore complete than before. This report gives a briefly basic knowledge of the bovine spongiform encephalopathy (BSE) to the public by clearly introducing the basic background, origin, events, policies and legislation relating to the bovine spongiform encephalopathy (BSE) worldwide. To the public, correctly choosing the lowest BSE-infected potential beef is much more important in the daily life. Through Figure 1 and Figure 2, the spongy degeneration in the brain caused by the bovine spongiform encephalopathy (BSE) can be easily understood. Through Figure 3, the geographical distribution of countries attacked by bovine spongiform encephalopathy (BSE) can be easily understood. Finally, correctly choosing the relevant productions from the non-BSE-infected areas rather than the BSE-infected areas is one of positive ways to avoid bovine spongiform encephalopathy (BSE).

\section{REFERENCE}

[1] Department for Environment Food and Rural Affairs, 2011. ARCHIVE: Bovine Spongiform Encephalopathy (BSE) [Online]. Available: http://archive.defra.gov.uk/foodfarm/farmanímal/disea ses/atoz/bse/index.htm [Accessed 22/12 2011].

[2] Department for Environment Food and Rural Affairs, 2009. ARCHIVE: BSE: Disease control \& eradication - Causes of BSE [Online]. Available: http://archive.defra.gov.uk/foodfarm/farmanimal/disea ses/atoz/bse/controls-eradication/causes.htm [Accessed 22/12 2011].

[3] United States Department of Agriculture, 2011.A Focus on Bovine Spongiform Encephalopathy [Online]. Available: http://web.archive.org/web/20080303135425/http://fsri o.nal.usda.gov/document_fsheet.php?product_id=169

[Accessed 22/12 2011].

[4] Food and Drug Administration Taiwan, 2009. The Risk Evaluation of Importing American Beef with Bone. Taipei: Department of Health Taiwan. 